\title{
Acknowledgement to Reviewers of Crystals in 2017
}

\section{Crystals Editorial Office}

MDPI AG, St. Alban-Anlage 66, 4052 Basel, Switzerland

Published: 10 January 2018

Peer review is an essential part in the publication process, ensuring that Crystals maintains high quality standards for its published papers. In 2017, a total of 381 papers were published in the journal. Thanks to the cooperation of our reviewers, the median time to first decision was 20 days and the median time to publication was 42 days. The editors would like to express their sincere gratitude to the following reviewers for their time and dedication in 2017:

Aakeröy, Christer B.

Abdellah, Kharicha

Abedi, Reza

Aboki, Tiburce A.

Abu Ali, Hijazi

Adhikari, Rajdeep

Adhikari, Ramesh

Admal, Nikhil

Agrosì, Giovanna

Ahmad, Rameez

Ahmed, Aymen A.

Akazawa, Housei

Akine, Shigehisa

Akitsu, Takashiro

Akm, Newaz

Alexander, Andrew

Alexandrov, Eugeny

Ali, Rejwan

Allan, Dave

Altomare, Angela

Alvarez-Alonso, Pablo

Alzina, Francesc

Aminorroaya Yamini, Sima

Andre, Baldermann

Andreea, Cojocaru

Ankireddy, Krishnamraju

Aoyagi, Kenta

Aquino, Manuel A.S.

Arabi, Hadi

\section{Lu, Luna}

Lubchenko, Vassiliy

Lube, Tanja

Lucchini, Mattia Alberto

Lucklum, Frieder

Lukac, Pavel

Luliński, Sergiusz

Luo, Zhenyue

Lupu, Nicoleta

Lusi, Matteo

Lyons, John L.

Ma, Hongan

Ma, Xiang

Maasilta, Ilari

Machida, Shinjiro

Madhavan, Vidya

Magasinski, Alexandre

Magdysyuk, Oxana

Mague, Joel

Mahata, Manoj Kumar

Mahjoub, Reza

Mahmudov, Kamran

Maia, Vergniory

Maisonneuve, Vincent

Makineni, Surendra Kumar

Malakooti, Mohammad

Maldonado, Stephen

Malecki, Jan Grzegorz

Maleky, Farnaz 
Arnault, Jean-Charles

Arnold, Donna C.

Arthur, Laganowsky

Artini, Cristina

Asenath-Smith, Emily

Atuchin, Victor V.

Aubert, Emmanuel

Aubry, J.P.

Aureliano, Manuel

Auriemma, Finizia

Avaro, Jonathan

Avarvari, Narcis

Babaee, Sahab

Badens, Elisabeth

Bag, Santanu

Bahr, David F.

Bailey, Scott

Baker, Robert

Balakrishnan, Geetha

Banfi, Francesco

Bank, Seth

Barnat-Hunek, Danuta

Barone, Paolo

Basilone, Luca

Baskoutas, Satirios

Batail, Patrick

Bauzá, Antonio

Bayraktar, Emin

Bayse, Craig A.

Beatrice, Perissutti

Beaudoin, Daniel

Beggs, Daryl

Bekheet, Maged F.

Belik, Alexei A.

Bellstedt, Peter

Belousov, Yury M.

Belyakov, Andrey

Belyakov, Vladimir

Benjamin, Wylie

Bérardan, David

Berdichevsky, Victor L.

Berdonosov, Petr S.

Berger, Reinhard
Malinauskas, Mangirdas

Malliakas, Christos

Malvić, Tomislav

Manciu, Felicia

Maniruzzaman, Mohammed

Manzano, Blanca R.

Mao, Yuanbing

Marcela, Mihai

Marcellini, Moreno

Marchetti, Fabio

Maresca, Francesco

Maria Del Pilar, Villar Castro

Marian, Jaime

Mariano, Paolo Maria

Martella, Daniele

Martín, Antonio E.

Martínez Pañeda, Emilio

Martinez, Ana

Martinez, Alejandro

Martire, Luca

Maruyama, Mihoko

Masatoshi, Tosaka

Masino, Matteo

Massi, Massimiliano

Massoud, Salah

Masu, Hyuma

Mathew, Nithin

Matson, Douglas M.

Matsumoto, Shoji

Matsumoto, Hiroshige

Matsumoto, Kazuhiko

Maurine, Montagnat

Mauro, Nicholas

Mautner, Franz A.

Maxwell, Gisele

Mazur, Ursula

Mazzotti, Matteo

McCabe, E.E.

McMillen, Colin

McNeil, Laurie E.

McQueen, Tyrel M.

Meakin, John D.

Mehdizadeh, Emad 
Bert, Nikolay

Bertness, Kris A.

Bertolotti, Federica

Besara, Tiglet

Beyerlein, Kenneth

Bhaskaran, Harish

Bhattacharia, Sanjoy

Bickermann, Matthias

Billinge, Simon

Birnie, Dunbar P.

Bisoyi, Hari Krishna

Björkman, Torbjörn

Blake, Sandy

Blancá, Eitel L. Peltzer Y.

Błaszczyk, Jarosław

Bloch, Witold

Bobrovsky, Alexey

Bocciarelli, Massimiliano

Boga, Carla

Bollani, Monica

Bon, Volodymyr

Borhani, Tohid N.G.

Bormashenko, Edward

Bos, Philip J.

Bos, Philip J.

Boualy, Brahim

Bouropoulos, Nikolaos

Boutinaud, Philippe

Bovornratanaraks, Thiti

Bradac, Carlo

Bradby, Jodie E.

Brandao, Paula

Brant, Jacilynn

Brazovskii, Serguei

Breedlove, Brian K.

Bridges, Craig A.

Brinck, Tore

Britton, T. Ben

Broer, Dick

Bronkhorst, Curt A.

Bruder, Enrico

Bruni, Giovanna

Brylew, Kamil
Meijide, Francisco

Melnikov, Gennady

Menčík, Jaroslav

Mercier, David

Merkulov, Oleg

Mesbah, Adel

Messerschmidt, Marc

Metere, Alfredo

Meyer, Franck

Meyer, Gerd

Micali, Norberto

Micallef, Aaron S.

Mikata, Yuji

Miletto, Ivana

Miller, Wolfram

Minakshi, Manickam

Minohara, Makoto

Minzioni, Paolo

Miranda, Perry

Miras, Haralampos N.

Miroshnichenko, Andrey

Miruszewski, Tadeusz

Mitov, Michel

Möckel, Robert

Modec, Barbara

Moghaddam, Narges Shayesteh

Mohamed, Sharmarke

Moiras, Haralampos

Monot-Laffez, Isabelle

Montalenti, Francesco

Mooers, Blaine

Mooibroek, Tiddo J.

Moonens, Kristof

Moore, Moreton

Moreno, Ignacio

Moron, Carlos

Morrison, Gregory

Mortazavi, Bohayra

Mortet, Vincent

Mosquera, Martin

Mosseri, Remy

Mota, Antonio J.

Mottillo, Cristina 
Brzeziński, Marek

Bučar, Dejan-Krešimir

Bueken, Bart

Bujak, Maciej

Burley, Jonathan C.

Bury, Wojciech

Bushuev, Yuriy G.

Byrne, Bernadette

Cadierno, Victorio

Cadot, Emmanuel

Calahorra, Yonatan

Camattari, Riccardo

Camerel, Franck

Cao, Dengke

CAPOBIANCO, AMEDEO

Capsoni, Doretta

Carlini, Riccardo

Carlucci, Lucia

Casañ-Pastor, $\mathrm{N}$.

Caselli, Alessandro

Casey, Sean

Cazorla, Claudio

Cazzanelli, Massimo

Ceretti, Monica

Cerny, Miroslav

Cerutti, Laurent

Cha, Judy J.

Chadeyron, Geneviève

Chae, Pil Seok

Chakoumakos, Bryan

Chakraborty, Indranil

Champagne, Benoit

Chan, Kwong Yu

Charman, William Neil

Chatterjee, Ruchira

Chavez-Angel, Emigdio

Chen, Daqin

Chen, Jhy-Der

Chen, Yuan-Tsung

Chen, Hongda

Chen, Hui-Yu

Chen, Xiang

Chen, Changfeng
Moussa, Sherif

Muhle, Claudia

Mukherjee, Soumya

Müller, Danny

Muñoz, Alfonso

Murray, Jane

Murugan, N. Arul

Musumeci, Chiara

Myong, Sua

Nadykto, Alexey B.

Nagano, Shusaku

Nakamura, Atsutomo

Nakano, Satoshi

Nalla, Venkatram

Nandi, Debaleena

Napoli, Gaetano

Naraghi, Mohammad

Narayanan Nair, Maya

Nardini, Marco

Nemec, Ivan

Németh, Péter

Nesterov, Dmytro

Nestola, Fabrizio

Neville, Suzanne

Neville, Frances

Newman, Janet

Nguyen, Minh Tho

Nicola, Lucia

Nicolotti, Orazio

Niemann, Hartmut $\mathrm{H}$.

Nieto, Fernando

Nishiura, Masayoshi

Nissinen, Maija

Nix, William

Norquist, Alexander

Norte, Richard

Novák, Igor

Novikov, Alexander

Novitskaya, Ekaterina

Oakhill, Jonathan S.

Oaki, Yuya

Ogawa, Naoki

Ogawa, Katsu 
Cheng, Hefeng

Cheng, Qian

Cheng, Zhenzhou

Cheng, Bing-Ming

Chern, Chyh-Hong

Chezganov, Dmitry

Chiang, Ming-Hsi

Chiappe, Daniele

Chierotti, Michele R.

Chikamatsui, Akira

Chiu, Kuan-Cheng

Choi, Young Jai

Choi, Duk-Yong

Choi, Sukwon

Choquesillo-Lazarte, Duane

Choudhary, Nitin

Christenson, Hugo

Christiane, Frank-Rotsch

Chu, Jiaxiang

Chu, Hailiang

Chuang, Wei-Tsung

Chung, Seok-Hwan

Chung, In

Cieslak, Jakub

Ciomaga Hatnean, Monica

Clark, Timothy

Clegg, W.J.

Clemens, Oliver

Cockayne, Eric

Cohen, Sidney R.

Collings, Ines E.

Comparelli, Roberto

Cook, Timothy R.

Cooper, Ryan C.

Cordill, Megan

Cordoyiannis, George

Cossairt, Brandi M.

Couce, María D.

Crist, Buckley

Croenne, Charles

Csetenyi, Laszlo J.

Cuberes, M. Teresa

Curtis, Neil F.
Oh, Hyunchul

Ohashi, Masashi

Ohfuji, Hiroaki

Ohkubo, Isao

Ohmagari, Shinya

Ok, Kang Min

Okada, Narihito

Okawara, Toru

Okumura, Hironori

Okumura, Yasushi

Olchowka, Jacob

Olejniczak, Iwona

Ortenzi, Marco A.

Ortuno, Manuel

Osgood, Richard

Ostadhassan, Mehdi

Oswald, Iain

Otten, Edwin

Ouerghi, Abdelkarim

Ouyang, Lizhi

Ozaki, Ryotaro

Paarmann, Alexander

Padrela, Luis

Pagan, Darren

Pagliara, Stefano

Pagola, Silvina

Pak, Y. Eugene

Pal, Rumpa

Palles, Dimitrios

Pan, S.L.

Panwisawas, Chinnapat

Papaefstathiou, Giannis S.

Papanikolaou, Stefano

Papanikolaou, Nikos

Parakhonskiy, Bogdan

Park, Jang-Ung

Park, Sangyoun

Pasán, Jorge

Paskaleva, Albena

Pastero, Linda

Pathak, Siddhartha

Pato Doldan, Breogan

Pawate, Ashtamurthy 
Da Silva, Joaquim C.G.E.

Da Venda Oliveira, Paulo José

Dabkowska, Hanna

Dadgar, Armin

Dahal, Tulashi

Daiguebonne, Carole

Dalconi, Maria Chiara

Dang, Jing-Shuang

Daniel, Beaton

Davidchack, Ruslan L.

Davis, Paul

Dawe, Louise

De Munno, Giovanni

De Nolf, Wout

De Roo, Jonathan

De Rosa, Claudio

Dedon, Liv R.

Dehzangi, Arash

Dell'Agli, Gianfranco

Delplace, Pierre

Demers, Hendrix

Derrick, Mott

Detz, Hermann

Dharavath, Srinivas

Dickens, Peter

Ding, Fei

Dippenaar, Rian

Dixon, David A.

Dong, Zhizhong

Dong, Faqin

Dong, Mingdong

Dong, Yalin

Doquet, Veronique

Dovesi, Roberto

Dreglea, Aliona Ivanovna

Dshemuchadse, Julia

Ebbinghaus, Stefan

Edagawa, Keiichi

Egberts, Philip

Egli, W.A.

Ehrler, Bruno

El-Azab, Anter

El-Gendy, Ahmed A.
Peckhaus, Andreas

Peña-Ruiz, Tomás

Penuelas, J.

Pereira, Andre

Perlepes, Spyros

Perry, Robin

Petrov, Ognyan E.

Petschek, Rolfe

Phan, Manh-Huong

Phung, Quoc Tri

Piepenbrink, Kurt

Pinto, Deesy

Pizzagalli, Laurent

Plyusnin, Victor F.

Podoliak, Nina

Poeppelmeier, K.R.

Polikanov, Yury

Polini, Riccardo

Pop, Alexandra

Porti, Marc

Posada, Chrystian

Potapov, Andrei

Potel, Sebastien

Pottlacher, Gernot

Poyet, Stéphane

Pradhan, Sangram K.

Prado-Gonjal, Jesús

Prasai, Binay

Prashanth, Konda Gokuldoss

Prehna, Gerd

Prestat, Eric

Price, Jason

Primc, Darinka

Primig, Sophie

Probert, Mike

Prochowicz, Daniel

Provis, John L

Prud'homme, Robert E.

Prywer, Jolanta

Psarobas, Ioannis

Ptasinski, Joanna

Pucci, Andrea

Pulati, Nuerxida 
Erba, Alessandro

Errandonea, Daniel

Escuti, Michael. J.

Eugenio, Fazio

Failla, Salvatore

Fakharuddin, Azhar

Falini, Giuseppe

Fan, Zhanxi

Fancher, Chris

Fanfrlik, Jindrich

Fanfrlík, Jindřich

Farhang, Faezeh

Fedin, Vladimir

Feitsma, Louris J.

Feldblyum, Jeremy I.

Felner, Israel

Feng, Xunda

Ferrando Soria, Jesús

Ficner, Ralf

Field, David

Filinchuk, Yaroslav

Finkler, Amit

Finney, Aaron

Finney, Eric E.

Fitch, Andy

Fleury, Blaise

Floerke, Ulrich

Forgan, Ross

Formela, Krzysztof

Fortenberry, Ryan

Francesconi, M. Grazia

Franco, Lourdes

Frank, Walter

Franke, Knut

Frazer, Laszlo

Fressengeas, Claude

Friedemann, Sven

Frisenda, Riccardo

Frontera, Antonio

Fuierer, Paul

Fujihara, Takashi

Fujishiro, Hiroki

Furia, Emilia
Puli, V.S.

Puli, Venkata

Punzo, Francesco

Pustan, Marius

Pyatnov, Maxim

Quinones, Rosalynn

Raabe, Dierk

Rabizadeh, Taher

Raffo, Antonio

Ragozin, Alexey

Rak, Zsolt

Ramire-Castellanos, Julio

Ran, Drori

Rao, Ashit

Raptopoulou, Catherine P.

Recarte, Vicente

Reddy, G.N. Manjunatha

Reddy, Babu J.N.

Reid, Joel W.

Rempel, Andrey A.

Ren, Guobin

Reyes-Gasga, José

Reynolds, William T.

Rezaei, Behrooz

Riccio, Michele

Richeton, Thiebaud

Richter, E.

Rigamonti, Luca

Rimoldi, Luca

Ringenberg, Mark

Rios-Torres, Ramon

Rishinaramangalam, Ashwin

Ritala, Mikko

Rizzato, Silvia

Rodenbücher, Christian

Rodriguez-Blanco, Juan Diego

Rodríguez-Hermida, Sabina

Rogl, Gerda

Rogl, Peter

Romero-Muñiz, Carlos

Roncal-Herrero, Teresa

Ronholm, Jennifer

Rosenblatt, Charles 
Fytas, George

Gabdulkhakov, Azat

Gal, Assaf

Galdamez, Antonio

Gallagher, John

Gallo, Katia

Gammer, Christoph

Gangopadhyay, Anup

Gao, Zhiqiang

Gao, Yunxiang

Gao, Weilu

Garbovskiy, Yuriy

Garcia-Belmonte, Germà

Garcia-Esparza, Angel T.

García-Martínez, Emilia

García-Miquel, H.

Garskaite, Edita

GASSIN, Pierre-Marie

Gaudry, Émilie

Gautier, Romain

Gavira, José

Gavira, José A.

Geijselaers, Hubert J.M.

Gelbstein, Yaniv

Génois, Romain

George, Fytas

Gersen, Henkjan

Gervais, François

Ghandi, Khashayar

Gharbi, Mohamed Amine

Ghetmiri, Seyed Amir

Giannakoudakis, Dimitrios

Giannici, Francesco

Gilbert, Mark

Gilioli, Edmondo

Gimenez-Pinto, Vianney

Girlando, Alberto

Giuffrida, Antonio

Glaum, Robert

Głowacki, Michał

Glushko, Oleksandr

Godinho, Jose R.A.

Goldberg, Israel
Ross, Kate

Roth, Johannes

Roy, Utpal N

Rudykh, Stephan

Ruffato, Gianluca

Ruthstein, Sharon

Ruzicka, Ales

Ryabchun, Alexander

Sadakane, Masahiro

Sadoc, Jean-Francois

Saha, Manik Lal

Sahul, Raffi

Sai, Hiroaki

Sainidou, Rebecca

Sakharova, Nataliya

Sakidja, Ridwan

Saleh, Ahmed

Salguero, Tina $\mathrm{T}$.

Salvador, James

Samsonenko, D.G.

Sanchez, Goar

Sánchez, Juan Francisco

Sánchez-Somolinos, Carlos

Sanchiz, Joaquin

Sangwan, Vinod K.

Santos, María Vila

Sasaki, Tomoyuki

Sasaki, Masahiko

Sato, K.

Sato, Hiroshi

Scepaniak, Jeremiah

Scheiner, Steve

Schenk, Anna S.

Schiavon, Marco A.

Schneider, Gerald J.

Schöllhorn, Bernd

Schowalter, Marco

Schreiber, Frank

Schulzke, Carola

Schwiegerling, Jim

Scott, James Floyd

Segado, Sergio Sanchez

Segvic, Branimir 
Golovin, Igor S.

Gómez-Aguirre, L. Claudia

Gomis-Bresco, Jordi

Goncalves, Antonio

Gordon, Wong

Gourdon, André

Grabowski, Sławomir

Graiff, Claudia

Grammatikopoulos, Panagiotis

Gránásy, László

Gratia, Paul

Green, David

Grigory, Volovik

Grishkovskaya, Irina

Grivel, Jean-Claude

Grombacher, Denys James

Gross, Kārlis-Agris

Groves, Matthew R.

$\mathrm{Gu}$, Jiande

$\mathrm{Gu}$, Qinfen

Guénolé, Julien

Guido, Todde

Guillon, Olivier

Guilmeau, E.

Guin, Tyler C.

Guo, Peijun

Gupta, Sanju

Gutiérrez-Urrutia, Iván

Hada, Masaki

Hadjikakou, Sotiris K.

Hagelueken, Gregor

Hagemann, Hans Rudolf

Halasyamani, P. Shiv

Hallsworth, J.E.

Hammer, René

Hammersley, Simon

Han, Kunwoo

Han, Yongsheng

Hanke, Michael

Hänschke, Daniel

Haque, M.A.

Harada, Takayuki

Hardiman, Mark
Seidel, Rüdiger W.

Seiner, Hanuš

Seisenbaeva, Gulaim A.

Seki, Takahiro

Sekine, Chihiro

Sekine, Akiko

Sellberg, Jonas

Senda, Miki

Seo, Dae-Shik

Seo, Yongbeom

Seppelt, Konrad

Serier-Brault, Hélène

Serra, Anna

Sevilla, Joaquin

Sewell, Thomas

Seydel, Tilo

Shabalin, Anatoly

Shabani-Nooshabadi, Mehdi

Shabib, Ishraq

Shahani, Ashwin

Shandarov, Vladimir M.

Shchyglo, Oleg

Sheldon, Matthew

Shenderovich, Ilya G.

Shestakov, Mikhail

Shevelkov, Andrei V.

Shi, Liyi

Shikata, Shinichi

Shim, Yong Gu

Shimpi, Manishkumar

Shiryaev, Andrei

Shrout, Thomas R.

Shyu, Jiin-Jyh

Siczek, Miłosz

Silke, Diedenhofen

Simpson, Garth J.

Singer, Sherwin

Singh, Gurvinder

Sinha, Abhijeet

Skapin, Sreco D.

Slageren, Joris Van

Slater, Peter Raymond

Ślebarski, Andrzej 
Harrington, James

Harrison, William

Hartel, Richard

Hashimoto, Tadanori

Hatzikraniotis, Euripides

Haughian, Karen

Hauser, Adam J.

Hawes, Chris S.

Hayashi, K.

He, Hongshan

Heine, Johanna

Helliwell, John

Hirasawa, Izumi

Hiroyuki, KOUSAKA

Hnida, Katarzyna

Ho, Shing

Hoch, Constantin

Holec, David

Holmberg, Vincent

Hong, Seung-Tae

Honma, Michinori

Höpfl, Herbert

Horgnies, Matthieu

Hosmane, Narayan

Hosono, Hideo

Houjou, Hirohiko

Hsu, Su-Wen,

$\mathrm{Hu}$, Wei-Ping

$\mathrm{Hu}$, Wenbing

$\mathrm{Hu}$, Huaiyuan

Huai, Baojuan

Huang, Cheng-Liang

Huczko, A.

Hung, Tai-Feng

Huo, Suguo

Ibáñez, Maria

Ikeda, Hiroshi

Ikehara, Takayuki

Im, Won Bin

Imai, Hiroaki

Imaz, Inhar

Imlau, Mirco

Ingrosso, Chiara
Sledzinska, Marianna

Slep, Kevin

Sleutel, Mike

Smeets, Stef

Sobolev, Vladimir

Solanki, Raj

Solarz, Piotr

Soluyanov, Alexey

Soma, Venugopal Rao

Somayazulu, Maddury

Son, Jae Sung

Song, Bohang

Sontakke, Atul D.

Soos, Zoltan G.

Spaepen, Frans

Spåhr, Henrik

Spina, Roberto

Stachowicz, Mateusz

Staley, Jonathan P.

Staszak, Katarzyna

Steinrück, Hans-Georg

Stellato, Francesco

Stojanoff, Vivian

Stotz, James

Stoumpos, Costas

Straumal, Boris B.

Stride, John Arron

Strzałkowski, Karol

Stumpe, Joachim

Sturman, Boris

$\mathrm{Su}$, Chie-Shaan

Su, Hai-Ching

$\mathrm{Su}$, An-Chung

Subbiah, Jegadesan

Suchomski, Christian

Suihkonen, Sami

Sumathi, R.R.

Sun, Haoran

Sun, Changquan Calvin

Sutherland, A.J.

Szeleszczuk, Lukasz

Szewczyk, Roman

Takagi, Youhei 
Isa, Fabio

Ishikawa, Tadahiko

Ishikawa, Ryuta

Islamoglu, Timur

Island, J.O.

Ito, Takeru

Ito, Hideto

Ivanov, Dimitri

Iwasaki, Takayuki

Jain, Sandeep K.

Jana, Debnarayan

Jang, J.I.

Janiak, Christoph

Janka, Oliver

Jankowska-Sumara, Irena

Jelsch, Christian

Jennett, Nigel

Jensen, Kirsten Marie Ørnsbjerg

Jeong, Nak Cheon

Jiang, Qinghui

Jirásková, Yvonna

Jo, Sae Byeok

Johnson, David

Johnson, Roger

Johnston, Karen

Joshi, Kaushik

Jung, Seungyong

Jung, Hyun

Jung, Yeonwoong

Jurado-Navas, A.

Jursich, Gregory

Kacher, Josh

Kaeswurm, Barbara

Kaganer, Vladimir M.

Kahr, Bart

Kakimoto, Koichi

Kalkan, A. Kaan

Kang, Youngsoo

Kang, Yvonne Qiongyue

Kapadia, Rehan

Kardum, Jasna Prlić

Karimov, Denis N.

Karouta, Fouad
Takagiwa, Yoshiki

Takahashi, Susumu

Takahiro, Kawamura

Takashima, Yohei

Tame, Jeremy

Tan, Gangjian

Tanaka, Kentaro

Tanatar, Makariy

Tang, Chungang

Tanner, Brian

Tanski, Joseph M.

Taras, Kolodiazhnyi

Taurino, Rosa

Tempesta, Gioacchino

Ten Kate, Melvin

Teranishi, Takashi

Tetean, Romulus

Thomas, P. John

Thomas, Altantzis

Tian, Hao

Tieu, Anh Kiet

Timofeev, Ivan V.

Tiribocchi, Adriano

Tiseanu, Carmen

Tkach, Alexander

Tobler, Dominique J.

Tochigi, Eita

Tokudome, Yasuaki

Tokumoto, Yuki

Tomoteru, Fukumura

Toney, Michael

Tongay, Sefaattin

Tourin, Arnaud

Travesset, Alex

Trivedi, Evan R.

Truster, Timothy

Tsai, Hui-Lien

Tsay, Chien-Yie

Tseng, Chih-Hua

Tsoutsouva, Maria

Tsutsumi, Jun'ya

Tu, Chi-Shun

Uda, Satoshi 
Kataeva, Olga

Katagiri, Hiroshi

Katsuki, Hiroyuki

Kauffeld, M.

Kawaguchi, Hiroyuki

Kawano, Jun

Keckes, Jozef

Kee, Hae-young

Keller, Sandro

Kennedy, John V

Kennedy, Kenneth

Khaliq, Jibran

Khan, Amir

Khansur, Neamul Hayet

Khare, Sanjay

Kidambi, Piran R.

Kieslich, Gregor

Kim, Yongho

Kim, Myoung-Jin

Kim, Haegyeom

Kim, Kyeong Kyu

Kim, Jae-Hoon

Kim, Yong

Kimura, Munehiro

Kimura, Shigeru

Kimura, Hideo

Kioseoglou, Joseph

Kirillov, Alexander M.

Kis, Viktoria K.

Kitazawa, Takafumi

Klein, Lisa C.

Klein-BenDavid, Ofra

Klosterman, Jeremy

Kobayashi, Atsuko

Kobold, Raphael

Kodama, Koichi

Koizumi, Haruhiko

Kojima, Kenichi

Kolář, Michal H.

Kolaric, Branko

Komar, Jarosław

Kondo, Hidemasa

Kondo, Mitsuru
Uemura, Kazuhiro

Uher, Ctirad

Uno, Kazuyuki

Unruh, Daniel

Uranga, Pello

Urbanski, Martin

Urbassek, Herbert M.

Urbonas, Liudvikas

Vakonakis, John

Vǎleanu, Mihaela Cristina

Valentini, Luca

Van Hecke, Kristof

Van Well, Natalija

Vaqueiro, Paz

Varga, R.

Vashist, Sandeep Kumar

Vaz, Carlos

Veprek-Heijman, Maritza G.J.

Verdejo, Begoña

Vergara, Alessandro

Vinnik, Denis

Vogt, Matthias

Voïtchovsky, Kislon

Von Freymann, Georg

Vouyiouka, Stamatina N.

Voyiadjis, George Z.

Vrcelj, Ranko

Wakamatsu, Takashi

Walia, Sumeet

Walker, Julian

Wallentin, Jesper

Walley, Stephen M.

Wallis, John D.

Wang, Pai

Wang, Yuejian

Wang, Haiyan

Wang, Ke

Wang, Haoyu

Wang, Hsin

Wang, Qijie

Wang, Xiaomu

Wang, Qisheng

Watauchi, Satoshi 
Kopel, Pavel

Kordesch, Martin E.

Kostakis, George

Kourkoumelis, Nikolaos

Koutselas, Ioannis

Krahl, Thoralf

Kravtsov, Victor

Kruszynski, Rafal

Kubicki, Maciej

Kubo, Kazuya

Kubo, Masataka

Kubota, Yasuhiro

Kukovec, Boris-Marko

Kumai, Reiji

Kuo, Dong-Hau

Kurahashi, Takuya

Kurganskaya, Inna

KURIMURA, Sunao

Kurlyandskaya, Galina V.

Kushwaha, Satya

Kuwata, Shigeki

Kuźnik, Nikodem

Kwan, Eugene E.

Kwon, Young-Wan

Kwon, Soon-Hong

Lafalce, Evan

Lagerlof, Peter

Lahoz, Fernando J.

Lamba, Doriano

Lambrecht, Daniel S.

Lambri, Osvaldo A.F.

Lang, Jian-Ping

Larsson, Per-Lennart

Laude, Vincent

Laukkanen, Pekka

Laurell, Fredrik

Laurenti, Marco

Lavrentovich, Oleg

Law, Stephanie

Lazanu, Sorina

Le Guennic, Boris

Le, Khanh Chau

Lee, Kang II
Watras, Adam

Weaver, Jordan

Weber, Cameron

Węglarz, Władysław P.

Wei, Qi-Huo

Wei, Zheng

Weinberger, Christopher

Welbourn, Chris

Wen, Jianguo

Wen, Jiajia

West, John L.

West, Anthony R.

Whatmore, Roger

Widdifield, Cory

Wierzchowski, Wojciech K.

Wilemski, Gerald

Wilkinson, Stephen

Williams, Jim

Williams, Oliver

Wilson, Justin J.

Wilson, Stephen

Wisniewski, Wolfgang

Wohlrab, Sebastian

Wolverson, Daniel

Wörle, Michael Dieter

Wormser, Maximilian

Wright, Jon

Wu, Jing-Yun

$\mathrm{Wu}$, Tao

Wu, Shin-Tson

Wuttke, Stefan

Wuu, Dong-Sing

Wyman, Ian

Xiao, Lizhi

Xiao, Shaoping

Xie, Weiwei

Xing, Feng

$\mathrm{Xu}$, Xiaoxue

$\mathrm{Xu}$, Wenyan

Xuefeng, Wang

Yamada, Yasunori

Yamakage, Ai

Yamamoto, Takashi 
Lee, Kyu Hyoung

Lee, Chih-Hao

Lee, Peter Peng Foo

Lee, Joonhee

Lee, Stephanie S.

Lee, Yun-Han

Lei, Wen

Lelidis, Ioannis

Lemaire, Martin T.

Lemmens, $P$.

Lemoine, Pierric

Lenert, Andrej

Leone, Philippe

Leou, Keh-Chyang

Létoublon, Antoine

Leyssens, Tom

Lezama, Luis

Lherbier, Aurélien

Lhuillier, E.

Li, Xiangping

Li, Kun

Li, Guoqiang

Li, Xiang

Li, Xingzhong,

Li, Ping

Li, Jiashen

Lightfoot, Philip

Lim, Hojun

Lin, King-Fu

Lin, Zekai

Lin, Ying-Chih

Lin, Youxi

Lin, Zheshuai

Lin, Shih-Yen

Lin, Ming

Lindquist, Robert

Lindqvist, Jan Erik

Ling, Florence T.

Ling, Bo

Linke, Dirk

Lisnevskaya, I.V.

Liu, Shyh-Jiun

Liu, Shengchun
Yamane, Hisanori

Yamato, Takehiko

Yanagida, Takayuki

Yang, Chen-I

Yang, Kesong

Yang, Shengfeng

Yang, Zhihua

Yang, Huaiyu

Yang, Yingchao

Yangui, Aymen

Yannopapas, Vassilios

Yaroshchuk, Oleg

Yates, Jonathan

Yates, Edwin A.

Yazdan, Nima

Ye, Enyi

Ye, Qun

Yeddu, Hemantha

Yin, Shi

Yingbo, Zhao

Yonemochi, Etsuo

Yoon, Tae-Hoon

Yoshida, Masaro

Yoshimatsu, Kohei

YOSHIO, Masafumi

You, Tae-soo

Young, Victor

Young, Kwo

$\mathrm{Yu}$, Zaiqun

Yu, Hak Ki

Yu, Qiang

Yu, Yang

Yu, Chin-ping

Zabotto, Fabio Luis

Zahn, Dirk

Zaikina, Julia

Zaitsev, Alexander

Zak, Alla

Zamanzade, Mohammad

Zangrando, Ennio

Zavalij, Peter

Zawadzka, Anna

Zbib, Hussein M. 
Liu, Wenlong

Liu, Tianbo

Liu, Shaobin

Liu, Yunling

Liu, Jun-Jen

Liu, Qiang

Liu, Chongyang

Lloret, Fernando

Lo, Ikai

Lobo, Rui F.M.

Łodziana, Zbigniew

Long, Stephen

Longobardi, Giorgia

Lorenz, Alexander

Los, Jan

Los, Przemyslaw

Löser, Wolfgang

Losilla, Enrique Ramírez

Lotnyk, Andriy

Lotz, Bernard

Lowe, Edward
Zhang, Guoqi

Zhang, Fajun

Zhang, Jingchao

Zhang, Yunyan

Zhang, Di

Zhang, HB

Zhang, Qichun

Zhao, Liuyan

Zheng, Xiaojia

Zheng, Kaibo

Zhou, Xiaozhou

Zhou, Yuanyuan

Zhou, Shuang

Zhou, Caizhi

Zielinski, Adam

Zografopoulos, Dimitrios

Zolotoyabko, Emil

Zotov, Nikolay

Zuo, Lijian

Zverev, Peter G.

(C) 2018 by the authors. Licensee MDPI, Basel, Switzerland. This article is an open access article distributed under the terms and conditions of the Creative Commons Attribution (CC BY) license (http://creativecommons.org/licenses/by/4.0/). 